\title{
Evaluación de estructuras MIMO sobre tecnología LTE de cuarta generación (4G) de telefonía móvil
}

\section{Evaluation of MIMO structures on fourth generation LTE technology for mobile networks}

\author{
Martin Diomedes Bravo ${ }^{1}$, Miguel Angel Sánchez ${ }^{2}$ y German Dario Vargas ${ }^{3}$
}

\section{RESUMEN}

El proyecto se encamino hacia la realización de un componente de software para la simulación de un sistema LTE que permitiera la evaluación de estructuras MIMO de diversidad y multiplexación. La modulación OFDM establecida para el enlace descendente fue la utilizada junto a las estructuras MIMO para su implementación en cada una de sus etapas. Se evaluó la capacidad del sistema para reducir los efectos del canal representado en desvanecimientos tipo Rayleigh, típico de un sistema selectivo en frecuencia. Esta evaluación se realizó a través de la tasa de error de bit (BER) versus la relación señal a ruido (SNR). De esta forma se pudo cuantificar el efecto que tienen los diferentes arreglos de antenas sobre el rendimiento del sistema, incluyendo estructuras MIMO que se encuentran fuera de los estándares actuales de la tecnología LTE y que dan un aporte valioso en cuanto al rendimiento bajo determinadas condiciones.

Palabras Claves: Rendimiento; 4G; LTE; MIMO; OFDM.

\begin{abstract}
The project is about the realization of software's component for the simulation of a LTE mobile telecommunication system, in which allows the evaluation of MIMO structures of multiplexing and diversity gain. This evaluation was conducted through the BER (Bit Error Rate) versus the SNR (Signal to Noise Ratio). In this way, it was possible to quantify the effect of different antenna arrays on performance system, including MIMO structures that are beyond current standards and LTE technology that could make a valuable contribution in performance of telecommunications systems under certain terms. The OFDM modulation was used with MIMO structures to simulate LTE physical layer. The simulation results validate the correct operation of the system and allow valuable conclusions about the performance of LTE as fourth generation (4G) of mobile telephony.
\end{abstract}

Keywords: LTE; OFDM; MIMO; Performance; $4 G$.

1 Ingeniero Electrónico, PhD. Universidad Surcolombiana. Grupo de investigación en Telemática - Neiva Av. Pastrana Borrero - Carrera 1. martin.bravo@usco.edu.co

2 Ingeniero Electrónico. Universidad Surcolombiana. Grupo de investigación en Telemática - Neiva Av. Pastrana Borrero - Carrera 1. svanegas.miguel@hotmail.com

3 Ingeniero Electrónico. Universidad Surcolombiana. Grupo de investigación en Telemática - Neiva Av. Pastrana Borrero - Carrera 1. gerdavao@hotmail.com 


\section{INTRODUCCIÓN}

El desarrollo de las telecomunicaciones en los últimos años ha contribuido en el mejoramiento de los servicios ofrecidos actualmente a los clientes; logrando que voz, datos y contenidos multimedia sean provistos con altos niveles de calidad. Los cambios que se vienen produciendo han influido de manera significativa en nuestras actividades diarias. En el caso de la telefonía móvil se pasó de contar con grandes terminales analógicos con un servicio de calidad moderada y baja cobertura a tener teléfonos móviles con múltiples servicios, reducido tamaño y una cobertura casi mundial, hasta llegar a convertirse en una herramienta fundamental en la vida cotidiana.

El gran desafío de las comunicaciones inalámbricas radica en la adversidad que el medio de propagación presenta a cualquier tipo de señal, lo que se manifiesta a través del "fading" o desvanecimiento, que degrada la calidad de la señal recibida. Para contrarrestar estos problemas son propuestas nuevas especificaciones de tecnologías y algoritmos de optimización, como por ejemplo, el uso de la tecnología MIMO (Multiple Input Multiple Output), la cual es proyectada para obtener ventaja de la propagación multi-trayectoria y conseguir de esta forma ganancia en diversidad y multiplexación, donde estos parámetros permiten garantizar un servicio de calidad al usuario final y optimización en el uso de espectro, respectivamente (Martin et al., 2009).

El termino cuarta generación (4G) hace referencia a un tipo de acceso móvil de banda ancha con una alta capacidad de red, conectividad a través de redes heterogéneas y alta calidad del servicio (QoS). El estándar 3GPP (3rd Generation Partnership Project) define la tecnología LTE (Long Term Evolution) como la evolución de la norma 3GPP UMTS (3G) (Wang et al., 2009). Una parte relevante en la tecnología LTE es la técnica de múltiples antenas llamada MIMO con el objetivo de aumentar la cobertura y las capacidades en la capa física. La tecnología MIMO (Multiple Input Multiple Output) proporciona a LTE la capacidad de mejorar aún más su rendimiento de datos y la eficiencia espectral que se obtiene con el uso de OFDM (Orthogonal frequency-division multiplexing). El sistema implementado permite caracterizar parte de la tecnología LTE; para la realización del mismo se usó la modulación OFDM, que se encuentra establecida para el enlace de bajada, implementado cada una de sus etapas y midiendo su desempeño bajo diferentes configuraciones de antenas.

Se han realizado diferentes investigaciones en busca de cumplir con los objetivos propuestos en las diferentes versiones de las especificaciones 3GPP. En (Furuskar et al., 2008) se presentan algunas de las características claves de la interfaz de radio LTE, incluyendo la capa física y recursos de radio, funciones de gestión, y su impacto en el rendimiento del sistema. En 2008 fue publicado el resultado de la investigación (Siomina et al., 2008) donde se mide la capacidad de una red LTE con usuarios ejecutando simultáneamente múltiples servicios, demostrando en el estudio que la diferenciación de tráfico y servicio priorizado son cruciales en un servicio con retardo crítico.

En (Maestro et al., 2008) se evalúan algunos algoritmos de planificación para SC-FDMA tanto en escenarios locales como amplios. Enmarcados en este contexto se decide evaluar el resultado y aporte de diferentes arreglos multiantenas en los sistemas inalámbricos de cuarta generación basados en tecnología LTE, desarrollando un componente de software que permita analizar la influencia de esta técnica en la redes de telefonía móvil. Otro trabajo interesante es el de (Ball et al., 2009), en el cuál se provee una comparación entre bucle cerrado y bucle abierto con estructuras MIMO para el proyectado OFDM, basado en el acceso de radio de banda ancha con tecnología 3GPP UTRA LTE, en este trabajo se concluye y demuestra que la tecnología MIMO es un método apropiado para mejorar el caudal de datos de los usuario especialmente en sistemas de mediana carga.

\section{METODOLOGÍA}

Esta investigación se realizó en los laboratorios de comunicaciones de la facultad de ingeniería de la Universidad Surcolombiana. Basados en investigaciones publicadas en el área de la telecomunicaciones de IEEE (Institute of Electrical and Electronic Engineering) sobre redes inalámbricas de última generación. 


\subsection{LTE}

LTE se postula como gran candidato a convertirse en estándar 4G. Basándose en un conjunto de tecnologías pensadas para que todas las comunicaciones de una red de telefonía móvil se originen mediante el protocolo IP que se usa en Internet. Esto implicaría que la totalidad del tráfico de la red de telefonía estaría compuesta por datos. Las características de LTE se pueden resumir en lo siguiente (Dahlman et al., 2007):

- Aumento de las tasas de datos de los usuarios, llegando a velocidades picos downlink y uplink de hasta 100 Mbps y 50 Mbps respectivamente.

- Reducción de los retrasos. Menor tiempo en establecer la conexión, reduciendo la latencia de transmisión.

- Reducción del costo por bit, lo que implica mejorar la eficiencia espectral.

\subsection{Modulación OFDM}

La multicanalización por división de frecuencias ortogonales OFDM surge como una alternativa potente para los esquemas de modulación convencional debido a su capacidad de transmisión de alta tasa de datos con una alta eficiencia de ancho de banda, implementación simple y robustez ante canales con desvanecimientos sel. En contraposición a lo que se ve en las típicas comunicaciones monoportadora o Single Carrier (SC) ilustrada en la figura 1-a, donde cada símbolo se transmite serialmente, es decir uno a la vez, ocupando todo el ancho de banda disponible; en una modulación multiportadora se envían los símbolos paralelamente en subportadoras adyacentes (Weste et al., 1998), usando la técnica de división de frecuencias. La actual tecnología OFDM es una evolución de FDM, el cual consistía en dividir la información en $N_{c}$ bloques. Cada uno de estos bloques seria multiplicado por una portadora diferente, con el propósito de reubicar los espectros de cada una de estas porciones en que ha sido dividida la señal, como se ilustra en la figura 1-b.

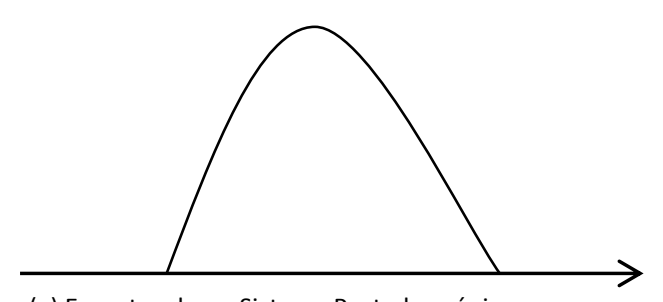

(a) Espectro de un Sistema Portadora única $\quad \mathrm{F}[\mathrm{H}$ >l
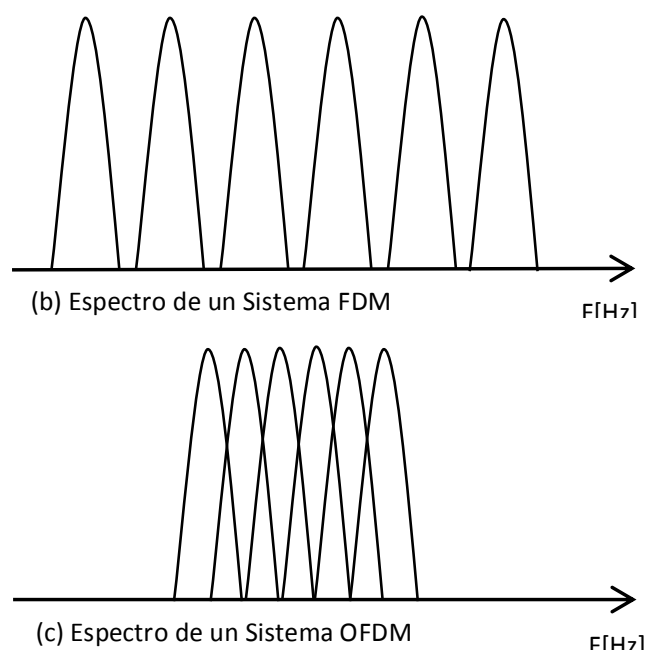

Fig. 1. Modulación monoportadora y multiportadora 
FDM representaba una alta ineficiencia espectral ya que requería de bandas de guarda para evitar la mezcla entre subportadoras que impidiera la recuperación de la información en el demodulador. Es por esto que aparece el concepto de portadoras ortogonales, el cual permite recuperar la información a pesar el traslape espectral que produce. A raíz de este concepto aparece como tal OFDM, el cual puede verse ilustrado en la figura 1-c. A nivel matemático si se usan $\mathrm{N}$ subportadoras y cada subportadora es modulada utilizando $\mathrm{M}$ símbolos, la señal OFDM es expresada en la Ecuación 1.

$$
v t={ }_{k=0}^{N-1} X_{k} e^{j 2 k n t / T}, 0 \leq t<T
$$

Donde $X_{k}$ representa los símbolos de datos, $\mathrm{N}$ es el número de subportadoras, y $\mathrm{T}$ es el tiempo del símbolo OFDM. El espacio entre las subportadoras $1 /$ T hace que los símbolos OFDM sean ortogonales entre cada periodo de símbolo.

\subsubsection{Etapas de la modulación OFDM}

Como se muestra en la figura 2, la señal de entrada de un sistema OFDM es una trama de datos en serie. Estos datos pasan a un formato de $\mathrm{n}$ bits de longitud de acuerdo al esquema de modulación utilizado. A la salida del mapeador se tiene símbolos ortogonales entre sí y a cada uno de ellos se les asignará una subportadora por medio de la IFFT. El conversor serie a paralelo organiza los datos para poder introducirlos a la IFFT y así generar los símbolos OFDM. La IFFT genera múltiples portadoras a diferentes frecuencias ortogonales dividiendo el ancho de banda disponible entre un número $N_{c}$ subportadoras. La trama de datos en paralelo sujeta al proceso IFFT constituye la modulación OFDM. La IFFT convierte un número de puntos de datos complejos de longitud $\mathrm{N}$ la cual es una potencia de 2 , en una señal en el domino del tiempo de igual número de puntos.

Después de esto se agrega el prefijo cíclico en cada símbolo para suprimir la interferencia intersímbolo (ISI) causado por la distorsión multitrayectoria. La figura 3 ilustra el proceso de adicción del prefijo cíclico. Se define la duración del PC (en número de muestras) por el entero $N_{p c}$ que debe ser mayor que la longitud de la respuesta al impulso del canal para evitar efectivamente ISI e ICI. Se copian y pegan al principio los $N_{p c}$ últimos bits del símbolo. Sin embargo La energía que se debe transmitir incrementa con la longitud del prefijo cíclico. La pérdida en SNR debida a la inserción del CP se puede calcular de acuerdo a la Ecuación 2.

$$
S N R_{\text {loss }}=-10 \log _{10}\left(\frac{N_{o}}{N_{o}+N_{p c}}\right)
$$

Donde $N_{p c}$ es la longitud del prefijo cíclico y $N_{o}$ la longitud original del símbolo OFDM.

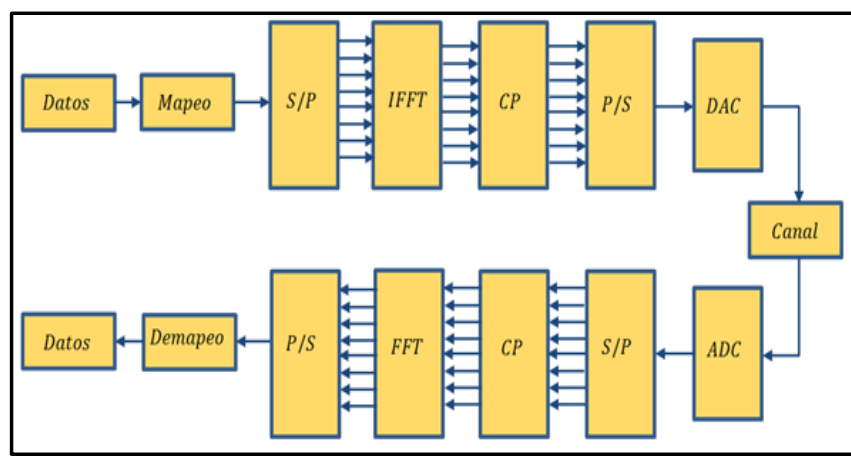

Fig. 2. Diagrama modulación OFDM

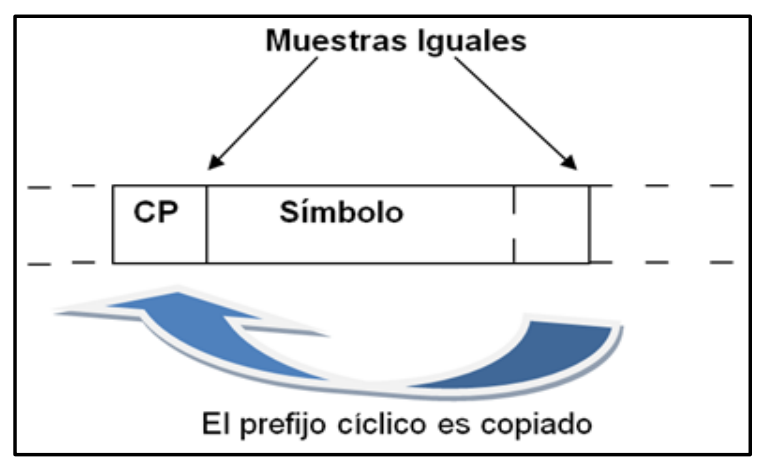

Fig. 3. Prefijo cíclico

En la práctica la señal OFDM se pasa por un conversor D/A para producir una señal análoga en banda base, la cual es modulada en RF para ser transmitida. 


\subsection{Canal inalambrico}

\subsubsection{Canal multitrayecto}

Una de las características fundamentales de las comunicaciones móviles es la propagación multitrayecto (multipath). Este tipo de propagación consiste en que entre el transmisor y el receptor existe más de un camino de propagación, ya que la señal radio que se propaga no es un rayo estrecho que va directamente de la antena transmisora a la receptora, sino un frente de onda amplio que se va difractando y reflejando a medida que encuentra obstáculos en su camino, como edificios altos, automóviles, montañas o irregularidades del terreno (Del Castillo et al., 2008). El fenómeno de la propagación multitrayecto se puede resumir diciendo que al receptor llegan varias réplicas de la misma señal, con diferentes retardos entre ellas.

\subsubsection{Modelos de desvanecimiento}

El fenómeno de desvanecimiento se suele describir estadísticamente mediante la distribución Rayleigh. La función de densidad de probabilidad (FDP) de una distribución Rayleigh está dada por la Ecuación 3.

$$
\operatorname{Pr}=\begin{array}{lr}
\frac{r}{\sigma^{2}} \exp -\frac{r^{2}}{2 \sigma^{2}}, & 0 \leq r \leq \infty \\
0, & (r<0)
\end{array}
$$

Donde $\sigma$ es el valor cuadrático medio (amplitud) de la señal recibida, $\sigma^{2}$ es su potencia media y $r$ la envolvente de la señal.

\subsection{Tecnologia MIMO}

Problemas como los desvanecimientos provocados por la propagación multitrayecto, pueden verse a través de técnicas como MIMO como una oportunidad para mejorar significativamente la capacidad y el rendimiento de los sistemas. Mediante el uso de múltiples antenas tanto en el transmisor como en el receptor. Estos canales pueden ser explotados para crear múltiples enlaces sobre la misma banda. Las técnicas de múltiples antenas pueden ser usadas tanto en transmisión como en recepción. Dependiendo la configuración que se tenga los sistemas MIMO se dividen en: MISO (Multiple-Input Single-Output), SIMO (Single-Input Multiple-Output), SISO (Single-Input SingleOutput) o simplemente como MIMO. La figura 4 ilustra los distintos arreglos.

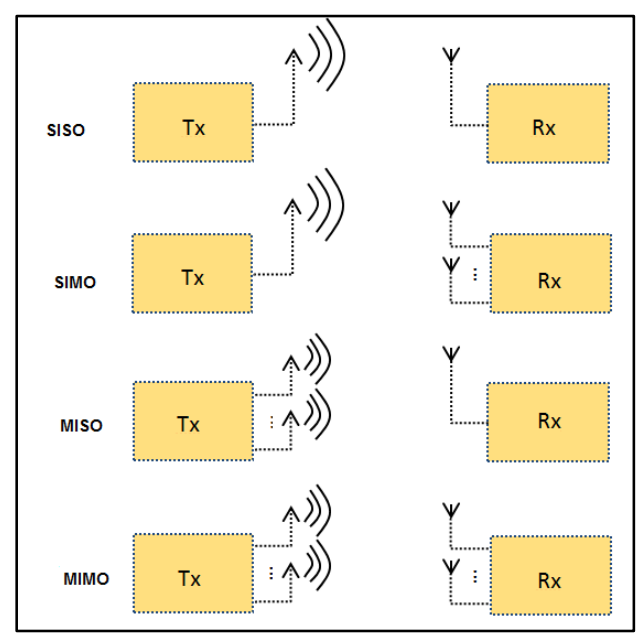

Fig. 4. Tecnologia de multiples antenas 


\subsubsection{Esquema de Alamouti}

El esquema de lazo abierto propuesto por Alamouti y que se encuentra ilustrado en la figura 5 consta de dos antenas transmisoras y una antena receptora que fácilmente se extiende al uso de varias antenas receptoras. Este esquema se conocería como código espacio-tiempo de bloques (STBC). La STBC proporciona diversidad espacial completa y hace uso de un algoritmo de decodificación sencillo que únicamente requiere de procesamiento lineal sobre las señales recibidas. La tabla 1 describe la secuencia de transmisión de Alamouti.

Tabla 1. Secuencia de codificación y transmisión de Alamouti

\begin{tabular}{|c|c|c|}
\hline Símbolos transmitidos & Antena 1 & Antena 2 \\
\hline Tiempo t & $s_{0}$ & $s_{1}$ \\
\hline Tiempo t + T & $-s_{1}{ }^{*}$ & $s_{0}{ }^{*}$ \\
\hline
\end{tabular}

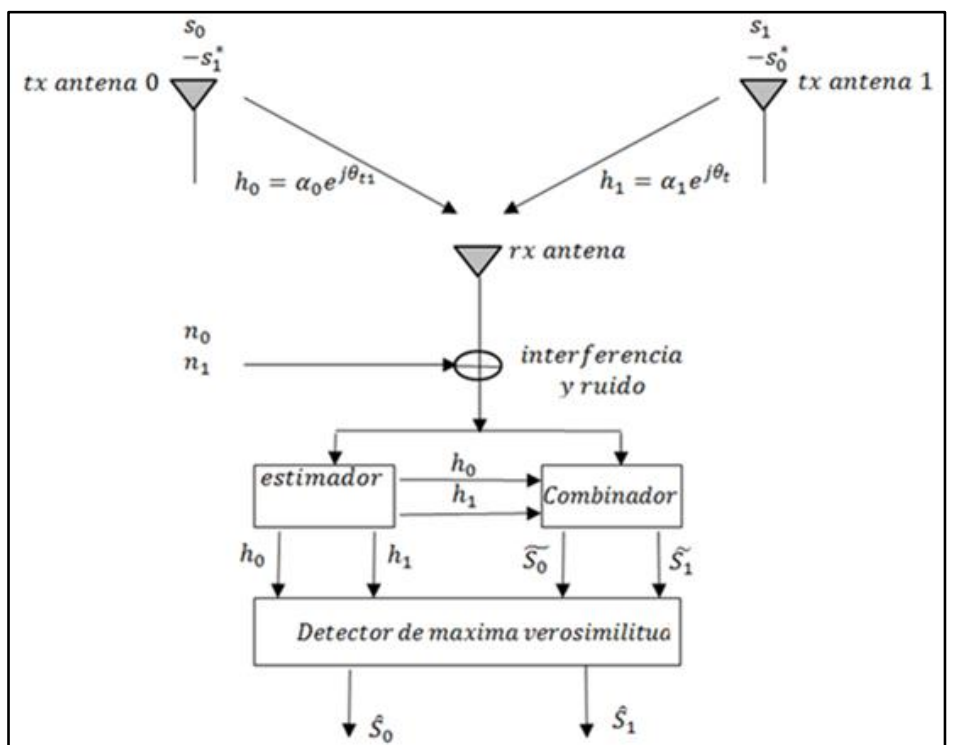

Fig. 5. Esquema de Alamouti de doble rama con un receptor. Tomado de (Alamouti, 1998).

Si se considera el canal en el instante $\mathrm{t}$ se puede modelar como una distorsión multiplicativa compleja Suponiendo que el desvanecimiento es constante a lo largo de dos símbolos consecutivos, se puede expresar como la Ecuación 4.

$$
\begin{aligned}
& h_{0}(t)=h_{0}(t+T)=h_{0}=\alpha_{0} e^{j \theta_{0}} \\
& h_{1}(t)=h_{1}(t+T)=h_{1}=\propto_{1} e^{j \theta_{1}}
\end{aligned}
$$

Donde $\mathrm{T}$ representa la duración del símbolo. Igualmente $\alpha_{1}$ y $\theta_{1}$ representan la amplitud y fase del canal. Conocido el canal, al igual que las señales transmitidas, podemos identificar las señales recibidas como se expresa en la Ecuación 5.

$$
\begin{gathered}
r_{0}=r(t)=h_{0} s_{0}+h_{1} s_{1}+n_{0} \\
r_{1}=r(t+T)=-h_{0} s_{1}{ }^{*}+h_{1} s_{0}{ }^{*}+n_{1}
\end{gathered}
$$

Donde $r_{0}$ y $r_{1}$ son las señales recibidas en el instante $\mathrm{t}$ y $\mathrm{t}+\mathrm{T}$ y donde $n_{0}$ y $n_{1}$ representan el ruido del receptor y las interferencias. El combinador produce las señales combinadas de la Ecuación 6 que posteriormente son enviadas al decisor:

$s_{0}=h_{0}{ }^{*} r_{0}+h_{1} r_{1}{ }^{*} \quad s_{1}=h_{1}{ }^{*} r_{0}-h_{0} r_{1}{ }^{*} \quad$ Ecu. (6) 
Donde $h_{i}$ representa la estimación del canal $h_{i}$. Sustituyendo se tiene la siguiente ecuación.

$$
\begin{aligned}
& s_{0}=\left(\alpha_{0}{ }^{2}+\alpha_{1}{ }^{2}\right) s_{0}+h_{0}{ }^{*} n_{0}+h_{1} n_{1}{ }^{*} \\
& s_{1}=\left(\alpha_{0}{ }^{2}+\alpha_{1}{ }^{2}\right) s_{1}-h_{0} n_{1}{ }^{*}+h_{1}{ }^{*} n_{0}
\end{aligned}
$$

Estas señales son enviadas al detector de máxima verosimilitud que, para cada señal $s_{0}$ y $s_{1}$, utiliza determinadas reglas de decisión.

\subsubsection{V-BLAST}

El proceso de transmisión en V-BLAST (Wolniansky et al., 1998) como se ilustra en la figura 6 consiste en dividir el flujo de datos inicial, de forma que quede repartido entre las antenas transmisoras. Posteriormente estos flujos se codifican en los símbolos de la constelación utilizadas, cada uno de estos flujos también llamados capas o layers son codificados de manera independiente y se transmiten simultáneamente en ráfagas en la misma banda de frecuencia. Estas antenas deben tener una separación mínima de una semilongitud de onda. Se asume por tanto, que se usa la misma constelación para cada subflujo y que las transmisiones se organizan en ráfagas de L símbolos. En V-BLAST el proceso de codificación es simplemente una operación de demultiplexación seguida de un mapeado independiente bit a símbolo de cada sub-flujo.

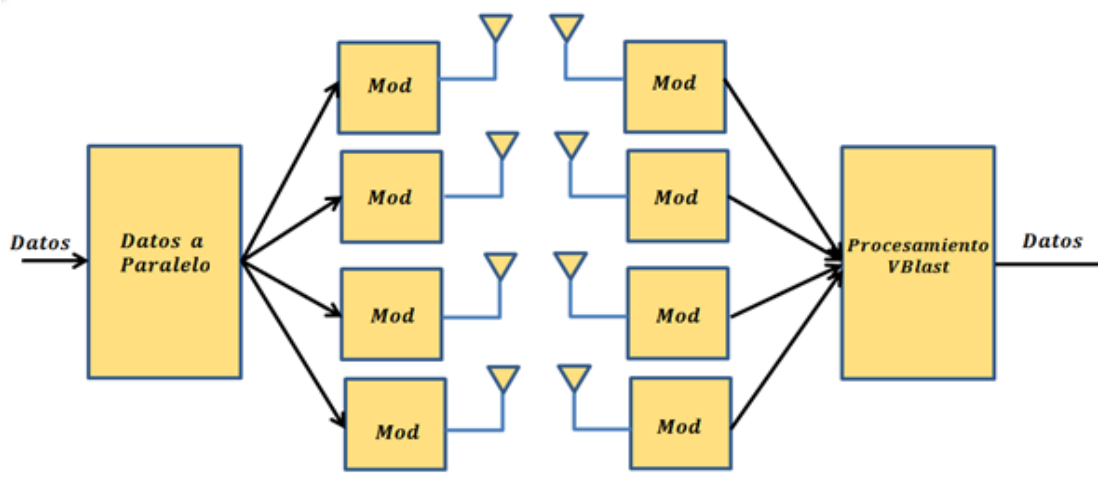

Fig. 6. Arquitectura V-BLAST

El algoritmo V-BLAST/MMSE consta de los siguientes pasos:

Fase de Inicialización

1. $i \leftarrow 1$

2. $\boldsymbol{G}_{1}=\boldsymbol{H}^{\dagger}$

4.

3. $k_{1}=\arg \min \quad \boldsymbol{G}_{1}{ }^{2}$

Fase de Recursión

6.

1. $\boldsymbol{w}_{k i}^{T}=\boldsymbol{G}_{i k i}$

2. $y_{k i}=\boldsymbol{w}_{k i}^{T} \boldsymbol{r}_{i}$

3. $s_{k_{i}}=Q\left(y_{k i}\right)$ 


\section{RESULTADOS}

Inicialmente se evaluaron las estructuras MIMO en solitario con el fin de comparar su rendimiento con diferentes tipos de modulación y configuraciones de antenas. Los parámetros de simulación se exponen en las tablas 2 y 3.

Tabla 2. Parámetros de simulación Alamouti

\begin{tabular}{|c|c|}
\hline \multicolumn{2}{|c|}{ Parámetros de simulación Alamouti } \\
\hline $\begin{array}{l}\text { Número de antenas } \\
\text { transmisoras }\end{array}$ & 2 \\
\hline $\begin{array}{l}\text { Número de antenas } \\
\text { receptoras }\end{array}$ & $1,2,3$ \\
\hline Esquema de modulación & $\begin{array}{l}\text { BPSK, QPSK, 16- } \\
\text { QAM }\end{array}$ \\
\hline Modelo del canal & Rayleigh \\
\hline Ruido & Gaussiano \\
\hline
\end{tabular}

Las figuras 7, 8 y 9 plasman los resultados obtenidos con el esquema de Alamouti para las diferentes configuraciones. Se evaluaron los arreglos de antenas $2 \times 1,2 \times 2$ y $2 \times 3$ de color rojo, azul y negro respectivamente, los graficos muestran que el aumento en el numero de antenas en receptor es proporcional a la disminucion del error. Igualmente el tipo de modulacion a utilizarse influye en el rendimiento de los diferentes esquemas.

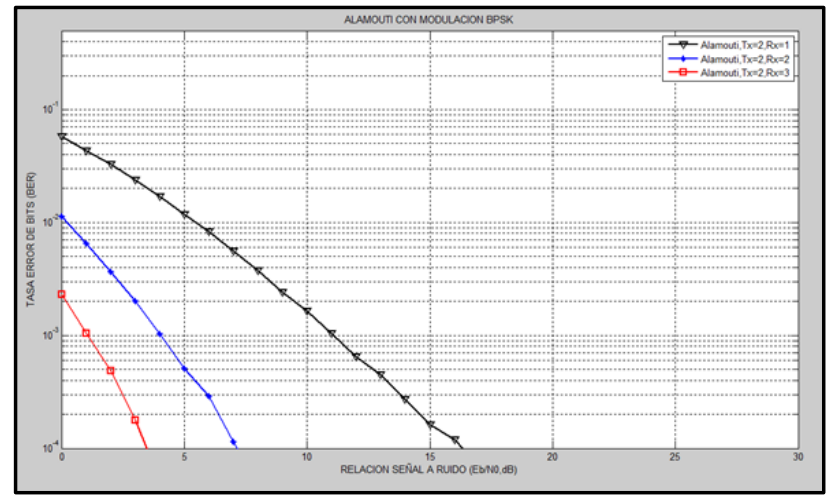

Fig. 7. Alamouti con modulación BPSK

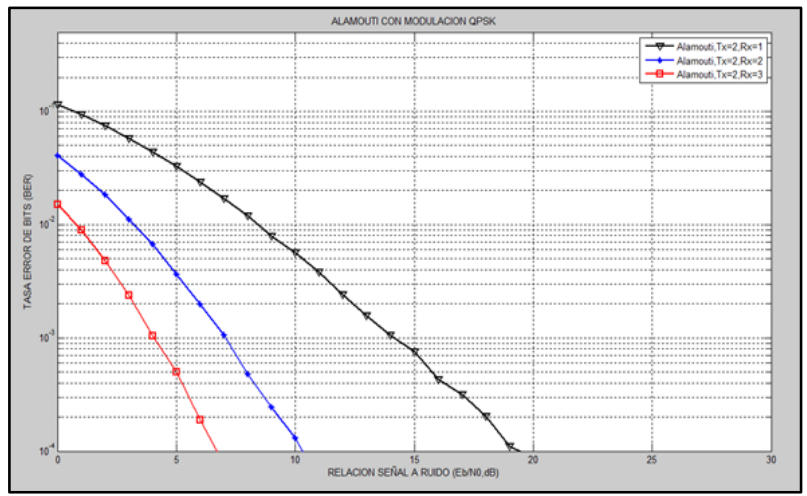

Fig. 8. Alamouti con modulación QPSK

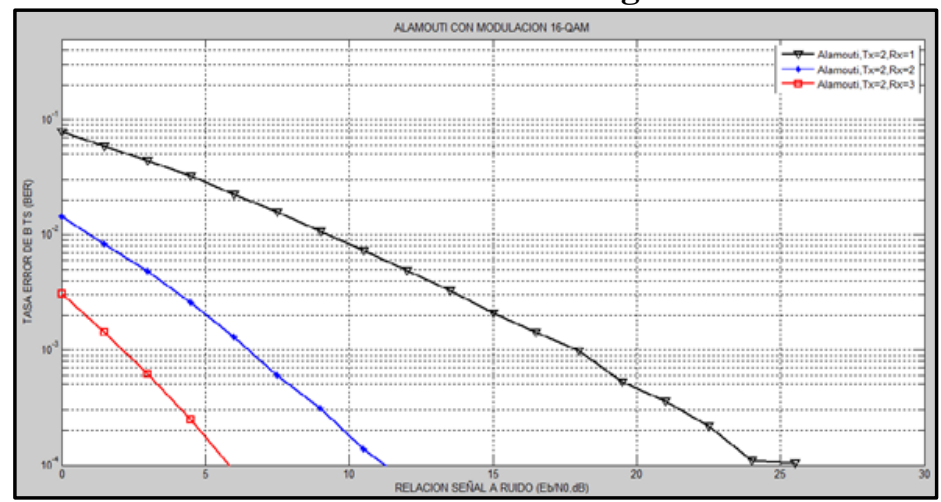

Fig. 9. Alamouti con modulación 16-QAM 
Tabla 3. Parámetros de simulación V-BLAST

\begin{tabular}{|c|c|}
\hline \multicolumn{2}{|c|}{ Parámetros de simulación V-BLAST } \\
\hline Tecnología MIMO & V-BLAST \\
\hline Detección & MMSE \\
\hline $\begin{array}{c}\text { Número de antenas } \\
\text { transmisoras }\end{array}$ & 2 \\
\hline $\begin{array}{c}\text { Número de antenas } \\
\text { receptoras }\end{array}$ & $2,3,4$ \\
\hline $\begin{array}{c}\text { Esquema de } \\
\text { modulación }\end{array}$ & BPSK, QPSK, 16- \\
\hline Modelo del canal & RAM \\
\hline Ruido & Gaussiano \\
\hline
\end{tabular}

Los resultados obtenidos con el uso de la tecnologia V-BLAST se plasman en las figuras 10,11 y 12 . La comparación entre las curvas muestra que la BER se mejora con el incremento en el número de antenas en recepción. Se destaca que la estructura con mayor rendimiento es la que posee 4 antenas en el receptor. La estructura $2 \times 2$ es la que se ve más afectada y esto se debe a que la arquitectura V-BLAST se puede ver muy influida por el canal. Cuando el número de antenas es igual en transmisión y recepción, en caso de haber un subcanal desfavorable puede afectar de manera significativa la BER.

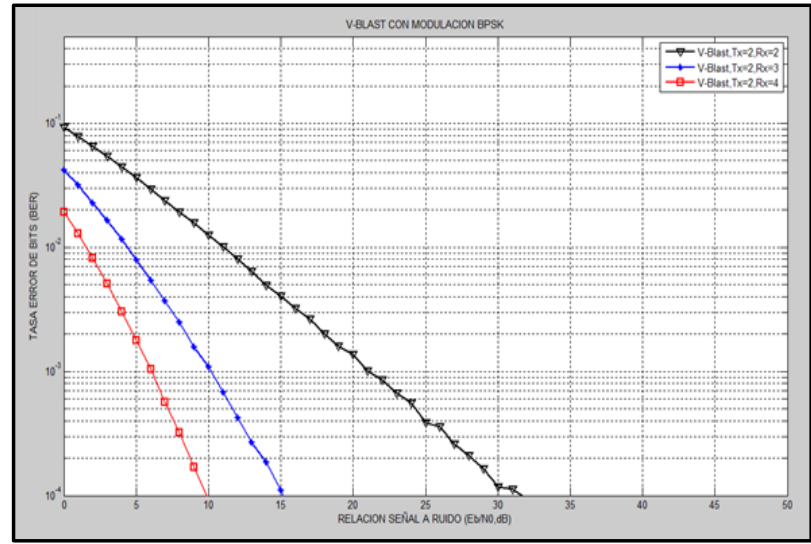

Fig. 10. V-BLAST con modulación BPSK



Fig. 11. V-BLAST con modulación QPSK

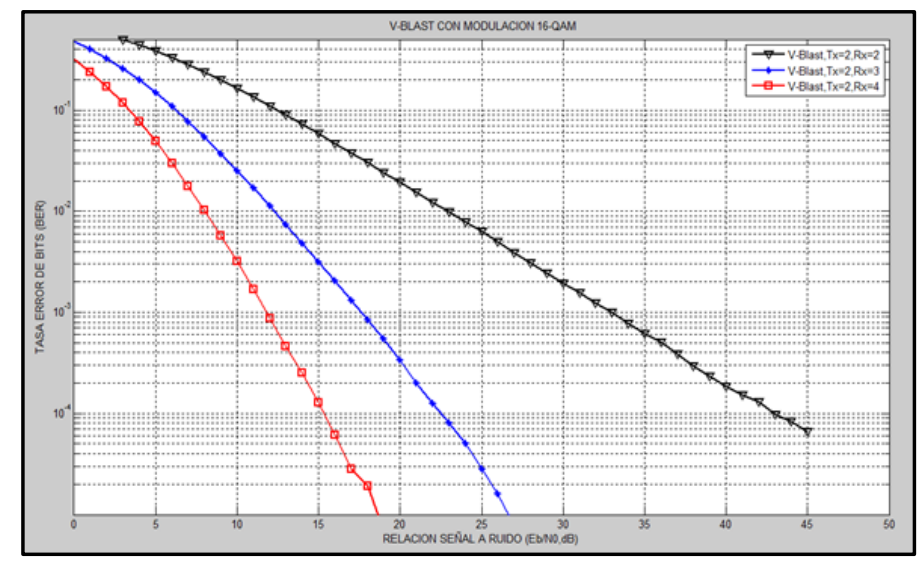

Fig. 12. V-BLAST con modulación 16-QAM 
Los arreglos 2x2 y 2x3 evaluados en V-BLAST presentan una mayor tasa de error, que cuando se evaluaron en Alamouti, esto se explica por la característica de redundancia en los datos que conlleva el esquema de Alamouti. El esquema V-BLAST está pensado para aumentar la eficiencia espectral sacrificando un poco la BER. Por el contrario los STBC como el esquema de Alamouti ofrecen mejores tasa de error que los esquemas de multiplexación como V-BLAST, incluso con un número menor de antenas. Lo cual era previsible al tratarse de codificaciones que aumentan la diversidad a través del número de antenas, sin aumentar la tasa de transmisión. A continuación se observan los resultados obtenidos al simular un sistema LTE que involucra tanto la tecnología MIMO como OFDM. La figura 13 representa el esquema de transmisión y recepción de un sistema MIMO-OFDM. Las figuras 14 y 15 correspondes al resultado usando el esquema de Alamouti y V-BLAST respectivamente.

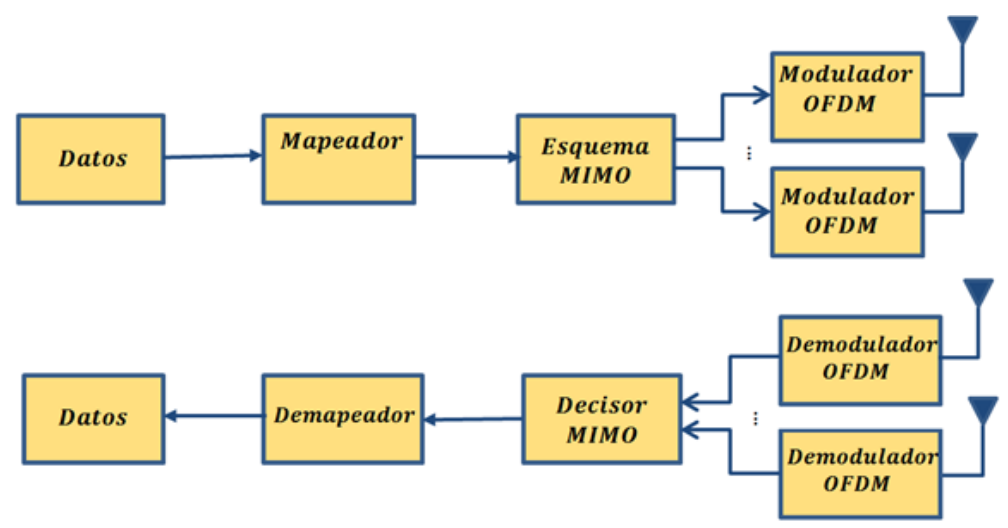

Fig. 13. Sistema MIMO-OFDM

La figura 14 afirma el efecto que tiene el aumento del número de antenas en recepción, siendo el esquema con 3 antenas en el receptor notablemente superior a los demás. Este sistema se caracteriza por presentar un nivel mayor de BER cuando se unen los esquemas MIMO con OFDM, que cuando se evalúan los arreglos MIMO en solitario. Esto se debe a que el uso de la modulación OFDM sobre canales selectivos en frecuencia sufre la desventaja de la pérdida de ortogonalidad entre portadoras debido al ensanchamiento Doppler. Debido también a que la tecnología OFDM tiene como propósito el aumento en las tasas de transmisión por medio de la optimización del espectro, en otras palabras de mejorar la eficiencia espectral, esto hace el sistema más propenso al incremento de la BER. Los resultados obtenidos en la figura 14 son levemente inferiores a los encontrados en (Chamkhia et al., 2012), esto se explica por el uso que hacen de estructuras pilotos con el fin de mejorar el rendimiento del sistema. Sin embargo los resultados no varían notablemente y concuerdan con los resultados obtenidos.

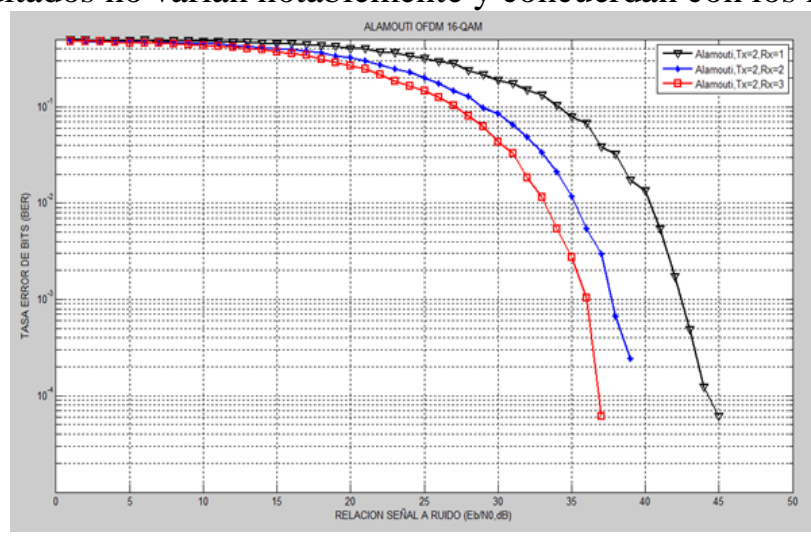

Fig. 14. MIMO-OFDM usando esquema de Alamouti

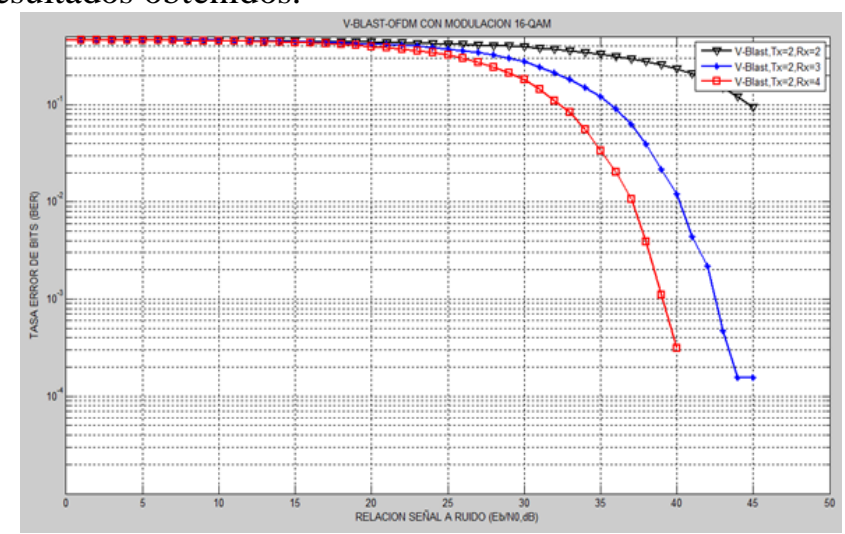

Fig. 15. MIMO-OFDM usando esquema V-BLAST

El resultado de la figura 15 indica que a pesar de tener el mismo comportamiento en cuanto al efecto del número de antenas en el rendimiento del sistema, es de destacar que el sistema usando el esquema V-BLAST presenta un nivel de BER mayor al presentado por el sistema cuando hace uso de la tecnología de diversidad, del esquema de 
Alamouti más exactamente. Esto era de esperarse si se tiene en cuenta los resultados que arrojaron estas estructuras cuando se evaluaron en solitario y donde el esquema de Alamouti obtuvo mejores prestaciones en cuanto a la reducción de la BER del sistema por su característica de diversidad. La configuración 2x2 es la que se ve sin duda más afectada con el aumento de la BER. La utilización de 3 y 4 antenas en el receptor mejora notablemente el rendimiento del sistema, por lo que se deduce la importancia de que el receptor tenga un mayor número de antenas que el transmisor, lo cual hace al sistema menos susceptible a condiciones desfavorables del canal.

La implementación de múltiples antenas en el receptor se ve limitada por las características de los equipos móviles, debido a que el aumento de las antenas conlleva que el dispositivo móvil aumente tanto su tamaño como su costo, sumándole a esto un consumo de energía elevado, causado en parte por los circuitos de selección o conmutación adicionales. Sin embargo esta limitante parece superarse con el desarrollo de materiales y dispositivos (Rivera, 2013) que hacen factible la implementación de múltiples antenas en los dispositivos móviles. El avance en nuevos métodos y técnicas ha llevado a considerarse factible el uso de arreglos de antenas tanto en transmisor como en receptor, tanto que 3GPP establece (Nakamura, 2009) para LTE Advanced arreglos 8x8 para el enlace de bajada y 4x4 para el enlace de subida. LTE Advanced es la propuesta para el desarrollo de la tecnología de cuarta generación y contempla planes de implementación y desarrollo hasta 2020.

\section{CONCLUSIONES}

El esquema de Alamouti y en general los STBC son una buena opción frente a la arquitectura V-BLAST en entornos ruidosos por su ganancia en diversidad. Se presentan como una solución atractiva cuando se necesitan sistemas de moderadas tasas de transmisión. Sin embargo, la arquitectura V-BLAST ofrece mayores capacidades en cuanto a datos, por lo que se considera una mejor opción en sistemas que exijan grandes tasas de transmisión.

La tecnología MIMO ofrece un incremento en las capacidades de los sistemas inalámbricos. En el presente trabajo se demostró las cualidades de los sistemas multiantenas con la implementación de diversos esquemas, las configuraciones mejoraron progresivamente el rendimiento del sistema con el aumento del número de antenas en el receptor, siendo el mejoramiento de la BER del sistema proporcional a la cantidad de antenas utilizadas.

Se ha demostrado que un sistema LTE que utiliza el esquema de Alamouti presenta un mejor comportamiento que uno basado en tecnología V-BLAST. Este comportamiento es medido con respecto a la tasa de error de bit (BER) y teniendo en cuenta las mismas condiciones de canal para ambas configuraciones. El esquema de Alamouti utilizado dentro de la tecnología LTE reduce la probabilidad de errores en el sistema, por lo que es recomendable su uso en entornos ruidosos.

En cuanto a las estructuras evaluadas en el proyecto se concluye que el aumento en el número de antenas en recepción está ligado directamente con la mejora en el rendimiento del sistema. Este principio aplica para todos las configuraciones de antenas evaluadas en LTE, excepto cuando se usa tecnología V-BLAST con el mismo número de antenas en transmisor y receptor, ya que el sistema se hace más susceptible a condiciones desfavorables del canal.

Las condiciones del canal influyen en la selección de los esquemas de modulación y codificación utilizados en la tecnología LTE. Cuando las distancias son largas y en condiciones severas, se utiliza un esquema de modulación robusto y menos propenso al ruido como BPSK, sacrificando la tasa de datos. Por otra parte cuando el canal se caracteriza por sus condiciones favorables se utilizan modulaciones como QAM, con los cuales se obtiene mayores tasas de transmisión. 


\section{REFERENCIAS BIBLIOGRÁFICAS}

Alamouti, S. 1998. A simple Transmit Diversity Technique for Wireless Communications, IEEE Journal on Selected Areas in Communications, vol. 16.

Ball, C.F. ; Mullner, R. ; Lienhart, J. ; Winkler, H. Performance analysis of Closed $\square$ and Open loop MIMO in LTE . Wireless Conference, 2009. EW 2009. European.

Chamkhia, H.; Omri, A.; Bouallegue, R. 2012. Improvement of LTE System Performances by Using a New Pilot Structure, International Journal of Wireless \& Mobile Networks, vol. 4.

Dahlman, E.; Parkvall, S.; Sköld, J.; Beming, P. 2007. 3G Evolution: HSPA and LTE for Mobile Broadband, Academic Press Publishing House.

Del Castillo, C. 2008. Estudio de la modulación OFDM y de los Efectos No Lineales Mediante Simulación en Matlab, Departamento de teoría de la señal y comunicaciones. Universidad de Sevilla.

Furuskar, A.; Jonsson, T.; Lundevall, M. 2008. The LTE Radio Interface-Key Characteristics and Performance, IEEE Communications.

Maestro, L.; Berardinelli, G.; Frattasi, S.; Mogensen, P. 2008. Channel-Aware Scheduling Algorithms for SC-FDMA in LTE Uplink, IEEE Communications.

Martin B. Obando, Walter C. Freitas Jr. and Francisco R. P. Cavalcanti, T. 2009. Cross-Layer Algorithm Using Hybrid MIMO Scheme for Video Transmission over Wireless Systems. XXVII Simposio Brasileiro de Telecomunicacoes - SbrT 2009, Blumenau.

Nakamura, T. 2009. Proposal for Candidate Radio Interface Technologies for IMT-Advanced Based on LTE Release 10 and Beyond (LTE-Advanced), $3^{\text {rd }}$ Workshop on IMT-Advanced.

Rivera, A. 2013. Gain Enhancement in Microstrip Patch Antennas Using Hybrid Substrates, Antennas and Wireless Propagation Letters, IEEE, vol. 12.

Siomina, I.; Wanstedt, S. 2008. The Impact of QoS Support on the End User Satisfaction in LTE Networks with Mixed Traffic, Ericsson Research.

Wang, H.; Lisimachos, P.; Luthra, A.; Song, C. 2009. 4G Wireless Video Communications, John Wiley \& Sons.

Weste, N.; Skellern, D. 1998. VLSI for OFDM , IEEE Communications Magazine, p. 127 - 131.

Wolniansky, P.; Foschini, G.J; Golden, G.D.; Valenzuela, R.A, 1998. VBLAST: An Architecture for Realizing Very High Data Rates Over the Rich-Scattering Wireless Channel, Proc. ISSE, Pisa, Italia. 